\title{
Development of Infrared and Millimeter Wave Scene Generators for the P3I BAT High Fidelity Flight Simulation
}

\author{
Jeremy R. Farris and Marsha Drake \\ System Simulation and Development Directorate, \\ U.S. Army Aviation and Missile Research and Development Center, \\ U.S. Army Aviation and Missile Command, \\ Redstone, Arsenal, Alabama 35898 \\ Jeremy.Farris@rdec.redstone.army.mil \\ Simulation Technologies, Inc., \\ 3307 Bob Wallace Avenue, \\ Huntsville, Alabama 35805 \\ Marsha.Drake@rdec.redstone.army.mil
}

\begin{abstract}
The Pre-planned Product Improvement (P3I) Brilliant Anti-Armor BAT submunition High Fidelity Flight Simulation (HFS) is an all-digital, nonreal-time simulation that combines the P3I BAT Six-Degrees-of-Freedom (6DOF), a high fidelity dual mode seeker model, tactical flight software, and infrared (IR) / millimeter-wave (MMW) scene generation to produce a tool capable of system design, algorithm development and performance assessment. The HFS development methodology emphasizes the use of high fidelity IR and MMW imagery so that the seeker algorithms / tactical software can be incorporated to form a Software-In-The-Loop (SWIL) simulation. The IR and MMW scene generators use validated IR and MMW target signatures with correlated IR and MMW backgrounds to produce a realistic scene for a variety of battlefield scenarios. Both the IR and MMW scene generators have evolved with the availability high performance computational capability and system development to meet the design and performance assessment requirements of the P3I BAT program.
\end{abstract}

\section{Introduction}

The Pre-planned Product Improvement (P3I) Brilliant Anti-Armor BAT submunition High Fidelity Flight Simulation (HFS) is being developed by the U.S. Army Missile Research, Development, and Engineering Center (MRDEC), Systems Simulation Development Directorate (SS\&DD) and the U.S. Army Tactical Missile System (ATACMS) / BAT Project Office support contractor as a tool to support system design evaluation, tactical algorithm development and performance assessment of the P3I BAT system. The HFS combines the P3I BAT 6-DOF model, a high fidelity dual mode seeker model, tactical flight software, and infrared (IR) / millimeter-wave (MMW) scene generation into an integrated, all-digital, Software-In-The-Loop (SWIL) simulation. As part of the P3I BAT suite of simulations, the HFS will be used to evaluate, analyze and assess the performance of the P3I BAT system. 
Along with being a standalone analytical tool, the HFS is the all-digital companion tool for the Hardware-in-the-Loop (HWIL) simulation and flight testing, allowing for extended scenario capabilities including additional targets and countermeasures. The HFS development methodology emphasizes the use of high fidelity IR and MMW imagery and tactical flight software-in-the-loop to maximize the commonalities between the HFS, HWIL, and tactical submunition. This greatly simplifies verification and validation $(\mathrm{V} \& \mathrm{~V})$ activities, and places a strong emphasis on IR and MMW scene generation.

This paper addresses the IR and MMW scene generators currently being used in the P3I BAT HFS, and how they have evolved with the availability of high performance computational capability and system development to meet the design and performance assessment requirements of the P3I BAT program.

\section{Background}

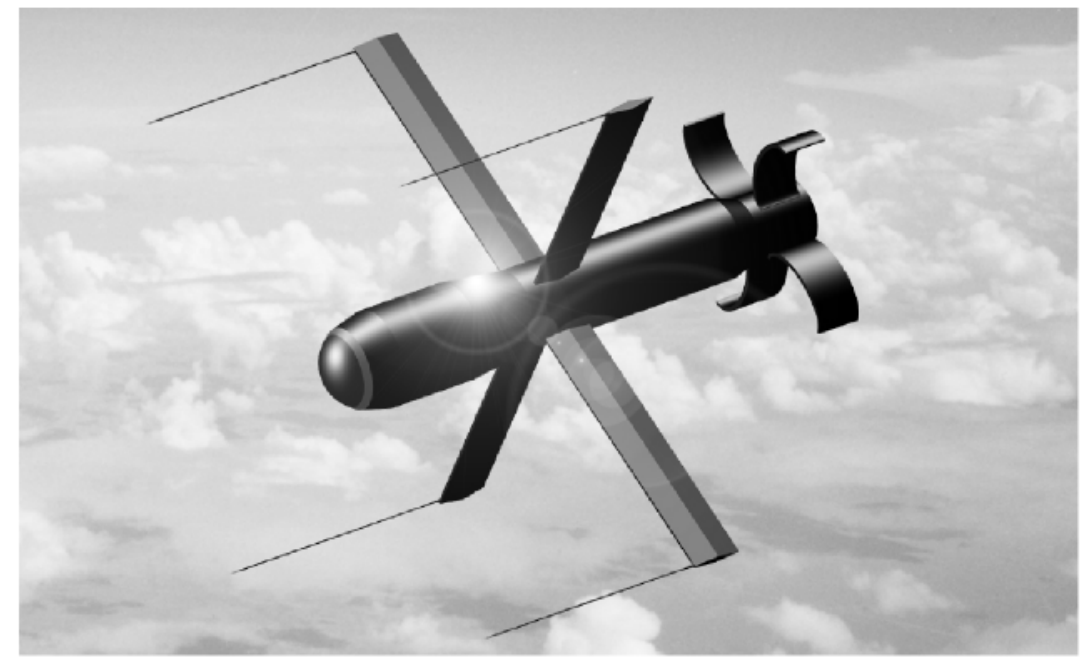

Fig. 1. P3I BAT Submunition

\section{P3I BAT Submunition}

The P3I BAT is the Pre-Planned Product Improvement for the Base BAT submunition (Fig. 1). The BAT and P3I BAT systems, after being dispensed from the host vehicle such as the Army Tactical Missile System (ATACMS), perform as autonomously guided smart submunitions that are designed to search, detect, and defeat armored vehicles in many weather conditions. The Base BAT submunition whose primary mission is to attack moving armored target arrays, initially utilizes acoustics to determine a general location of the target, then employs an IR seeker for acquisition 
and terminal homing. The P3I BAT submunition has the same physical characteristics as the Base BAT configuration, but incorporates an improved dualmode (IR/MMW) seeker design and an improved warhead that allows it to engage soft targets. These features greatly expand the possible engagement environments and target set. [1]

\section{HFS}

The P3I BAT HFS is a high-fidelity closed-loop simulation of the P3I BAT submunition that begins at dispense out of the carrier vehicle, and ends at submunition impact on the target. The methodology for the HFS development is primarily based on the methodology previously developed for the Javelin Antitank weapon system Integrated Flight Simulation (IFS). [2, 3] One of the major differences between these systems lies in the dual mode IR/MMW seeker used by the P3I BAT submunition. The Javelin seeker is an earlier generation IR-only seeker, and as such was not as complex to simulate and analyze as the dual mode P3I BAT seeker. To date, there are no other known dual mode simulations that approach the HFS in complexity and fidelity. [1]

The HFS was originally proposed as a tool to support the downselect between two competing seeker developers for the P3I BAT submunition. By integrating the seeker developer's detailed seeker model and algorithms into the HFS environment, the performance of each seeker design could be analyzed, and the effects of the seeker design on overall system performance could be quantified. The role of the HFS has since been expanded to include many areas, including providing the one (submunition)-on-many (targets) performance capability as an input to the many (submunitions)-on many (targets) system effectiveness simulation, STRIKE. [1]

\section{Methodology}

The HFS development methodology emphasizes four primary areas:

1. Reuse of Base BAT subsystem models common to P3I BAT

2. Use of tactical flight software-in-the-loop

3. Maximize use of common subsystem models between the HFS and HWIL.

4. Use of validated IR imagery and MMW measurement data for targets and backgrounds.

The primary goals behind the methodology are to:

1. Reduce development time.

2. Simplify verification and validation activities.

3. Provide an accurate assessment of the system's performance.

This approach to simulation development requires that the IR and MMW scene generators incorporate high fidelity target and clutter models, and the dual mode processing within the seeker requires that the MMW target and clutter models be 
generated in registration with the corresponding IR model. The use of high fidelity facet target models and backgrounds for the generation of IR imagery was a proven simulation methodology under the Javelin program. However, the critical test for the development of the P3I BAT HFS was to explore whether millimeter wave radar "imagery" could be incorporated into the simulation and utilized at the same time as the infrared imagery, with the same good results as those obtained using IR-only. No information was available as to whether this idea was feasible. Discussions were held with members of the MMW Longbow Hellfire Modular Missile System (LBHMMS) HWIL simulation team to obtain their expert opinions on the feasibility of the idea. They provided considerable information on the procedures and processes used to simulate MMW targets and clutter using the HWIL MMW radar arrays in real time. After these discussions, it was determined that these real-time HWIL modeling processes could be adapted and merged with IR clutter techniques to be run in a nonreal-time all digital simulation. [1]

\section{Model Development}

The P3I BAT HFS IR and MMW scene generation development has evolved with the availability of high performance computational capability and the requirements of the P3I BAT program. To fully appreciate this evolution, the fidelity of the IR and MMW target and clutter models will be discussed along with rendering techniques applied for image generation.

\section{IR Target Models}

Under the Javelin program, the U.S. Army MRDEC Missile Guidance Directorate (MGD) developed a methodology for applying IR target measurement data to 3 dimensional target facet models. A target geometry model is developed with "enough" polygons to represent the resolution of the calibrated IR imagery. The vertices are then "painted" with the collected measurements and rendered applying gouraud shading. This target development and rendering methodology has proven to be a valid approach on the Javelin missile programs, and was chosen for the P3I BAT IR scene generator.

As the program matured and the P3I BAT HWIL facility was developed, it became evident that the computational load to render the high polygon target models would not meet the HWIL real-time requirements. So, the HWIL personnel developed a methodology to decimate the number of facets, and apply texture(s) that would adequately represent the resolution of the calibrated IR imagery. (see Fig. 2) [4]

Although the high polygon targets were not as much of an issue for the non-realtime HFS, the availability of low-cost high performance Silicon Graphics capable of rendering 12 bit textured polygons made the incorporation of algorithms to render the HWIL real-time targets possible, and the advantages offered by the approach include improved scene generator efficiency and assures commonality with HWIL, reducing cost and streamlining the verification and validation process. 


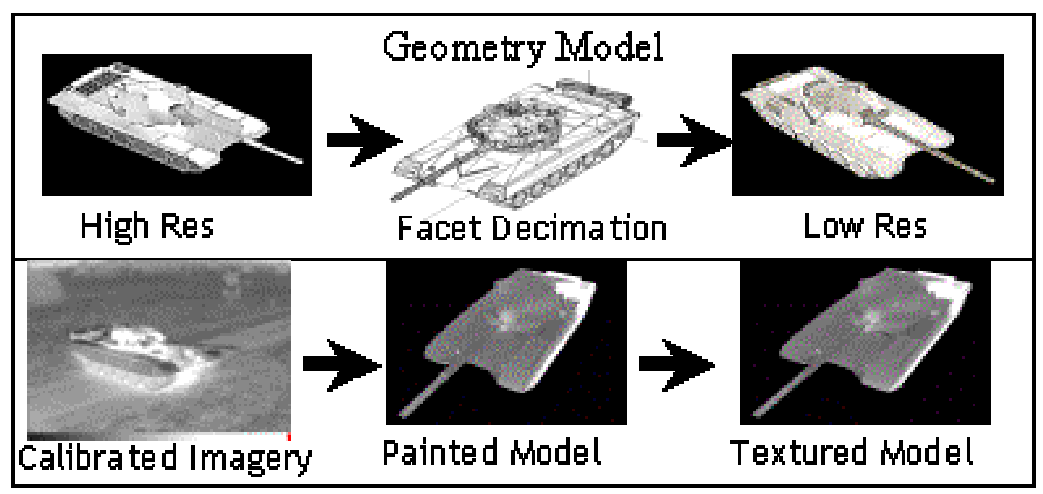

Fig. 2. P3I BAT HWIL Target Development Methodology [4]

\section{MMW Target Models}

Based on the LBHMMS HWIL experience, point scatterer models were developed for the P3I BAT program. The MMW target models developed have 150 scatterers per polarization per 0.5 degrees in azimuth and 5 degrees in depression. Each model has been verified and validated by comparing model frequency-averaged RCS and HRR aim-point statistics to those from the original measurement model [5]. The models are registered to the IR model by correlating the scatterer locations geometrically to the IR target coordinate frame. (see Fig. 3). [4]

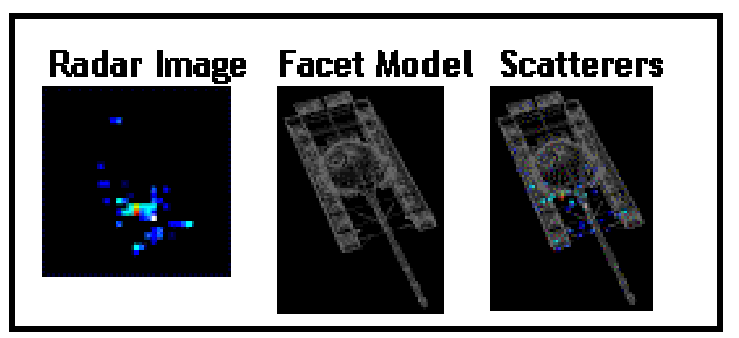

Fig. 3. P3I BAT MMW Target Development Methodology. [4]

In the utilization of these models, the number of scattering bins generated in target model development can approach 2,400 per polarization and more than 100 scattering centers thresholded from these bins are typically chosen. The computational requirements to process these scatterers are quite significant and a study has been made to quantify the effects of reducing the number of scattering centers to reduce run times and meet HWIL real-time requirements. Preliminary results indicate that the reduction of scatterers by half will generate RCS and aim-point statistics that are within the acceptable range of measurement statistics [5]. This reduction along with conventional parallel processing techniques will allow the HFS MMW scene generator to simulate complex battlefield scenarios within a reasonable amount of runtime. 


\section{IR Clutter Models}

Under the Javelin program, the U.S. Army MRDEC Missile Guidance Directorate (MGD) developed a methodology for applying IR clutter measurement data to a class map describing the site to be simulated. Each class map consists of description of the features of the site and typically consists of 1-foot increment descriptions, with the classes characterized as shown in Table 1.

Table 1. Class Map Descriptions

\begin{tabular}{c|c}
\hline Class & Description \\
\hline 0 & Bushes \\
1 & Tall Grass \\
2 & Brush \\
3 & Short Grass \\
4 & Road Sand \\
5 & Normal Sand \\
6 & Powder Sand \\
7 & Sparse Short Grass \\
8 & Dirt \\
9 & Gravel \\
10 & Asphalt \\
11 & Medium Veg \\
12 & Mixed Forest \\
13 & Deciduous Trees \\
14 & Coniferous Trees \\
15 & Snow \\
16 & Road Snow \\
17 & Traveled Road Snow \\
18 & Snow over veg \\
19 & Rock \\
22 & Sky \\
59 & Shadow \\
\hline
\end{tabular}

Initially, the P3I BAT IR scene generator rendered the clutter as a polygon mesh with each vertex "painted" to represent the measured class temperature data. If topography data was available, each vertex elevation was modified appropriately. At one foot resolution, the time to render the background was quite significant, and efforts to improve the scene generation efficiency were investigated. Again, the availability of low-cost high performance Silicon Graphics capable of rendering 12 bit textured polygons made it possible to represent the clutter with fewer polygons and still maintain the resolution of the class map. This approach was also chosen by the HWIL facility and provides one more area of commonality between the HFS and HWIL simulation domains. 


\section{MMW Clutter Models}

The dual mode processing with the P3I BAT seeker requires that the MMW backgrounds be generated in registration with the corresponding IR model. This is controlled by the utilization of a class map (see IR Clutter Model description). Normalized sigma 0 values obtained from collected measurement data are used in conjunction with the corresponding class map to generate the normalized even and odd polarization clutter map. This is obtained by applying the statistics reported to:

$$
\sigma_{\mathrm{o}}(\text { random, normalized })=\sigma_{\mathrm{o}}(\text { normalized })+20 \log _{10} \mathbf{r}
$$

where $\mathbf{r}$ is a Raleigh distribution with a mean of 1. The user then computes the sine of the depression angle to the given clutter patch and applies:

$$
\sigma_{\mathrm{o}}=\gamma \sin \theta
$$

where $\theta$ is the depression angle and $\gamma$ is the normalized sigma0. This model is considered valid in the range of depression angles of interest to this program. [6]

Currently, the BAT P3I HFS MMW scene generator has the option to read in pregenerated clutter maps or to dynamically generate the clutter maps on a run-to-run basis. Depth buffer data obtained from the IR scene generator provides information on which cells are "shadowed" by the target, and the model generates a list of sigma0 values to be processed. As can be imagined, the computational requirements are significant and efforts to optimize clutter cell processing are underway. This includes investigating the application of HWIL real-time optimized vector processing techniques.

\section{Verification and Validation}

Verification and validation of the P3I BAT IR and MMW scene generators is an ongoing process. The approach of utilizing IR and MMW target models that are developed with collected, not predicted, data simplifies the process but still requires that each model is validated with the calibrated measurement data. On the P3I BAT program, the target and clutter model developers have established and implemented verification and validation procedures to the approval and satisfaction of P3I BAT simulation community. In addition, the techniques by which the scene generators generate the simulated battlefield have been proven valid on other programs. The utilization of HWIL and flight test data has been critical in the verification and validation process and the commonality between the HFS and HWIL scene generation has streamlined these activities. Fig. 4 shows an example comparison between HFS and test IR imagery. 

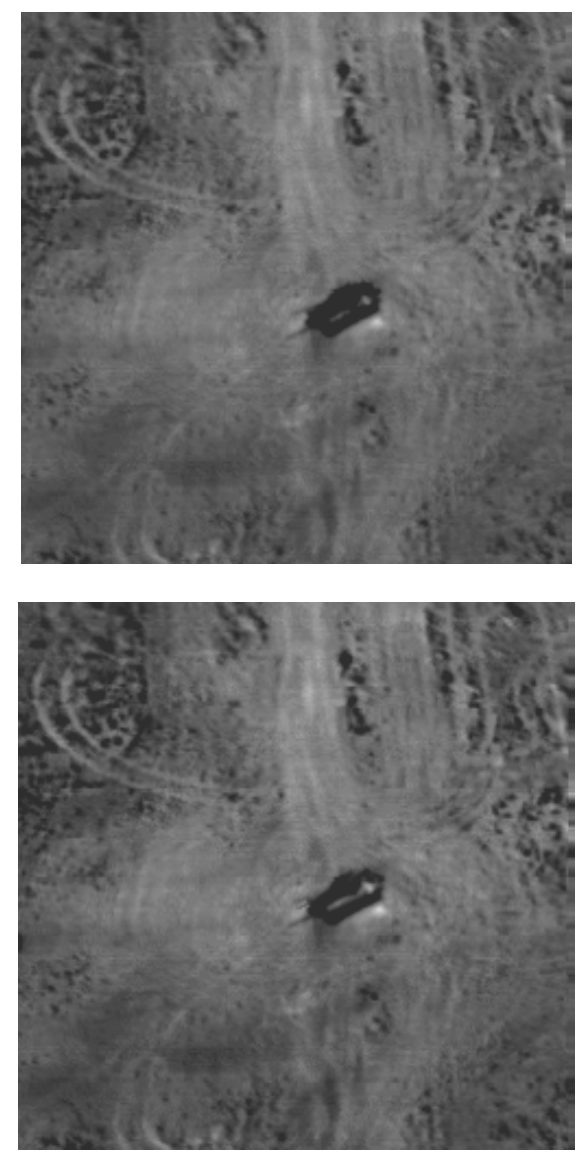

Fig. 4. P3I BAT Captive Flight Test Image (Top) and Simulated Image (Bottom)

\section{Current Utilization}

The P3I BAT HFS is currently being used in several studies. One study in particular is being performed to evaluate the tracker algorithms performance across a suite of target and clutter models. To support this study, upgrades to the IR and MMW scene generators were implemented to support the large Monte Carlo run matrices to be executed. One such upgrade has been proven to be instrumental in the timely execution of the analysis. Historically, IR and MMW clutter models are pregenerated and clutter variation is only obtained by reading in a new pre-generated model that is quite timely on a run-to-run basis. The P3I BAT signatures IPT developed a methodology in which collected class map statistics for particular sites, seasonal, and weather conditions were processed to form a clutter vector. For a Monte Carlo run set, both the IR and MMW scene generators utilize these clutter vectors to dynamically generate textures (IR) and normalized clutter maps (MMW) at 
the beginning of each run. In addition, a methodology was defined to apply a level term to the IR target model and allow for "yesterday's" target be placed in "today's" background. This capability provided the HFS an efficient means to Monte Carlo the IR and MMW clutter models, and allowed the evaluators to study the effects of clutter variation on the tracker algorithms.

\section{Future Enhancements}

There are several ongoing and planned activities to enhance the capabilities of the P3I BAT HFS IR and MMW scene generators. The activities are currently centered around increasing the fidelity and complexity of the simulated battlefield and implementing optimization techniques currently utilized in the HWIL simulation. Upgrades include the addition of discrete objects such as individual trees and buildings, and realistic target exhaust plume heating and obscuration effects. Battlefield effects such as burning targets and countermeasures (smoke, flares, etc.) will also be added as those models are developed.

In addition, the personnel responsible for the P3I BAT HWIL are currently responsible for developing a MMW scene generator for the Common Missile program that utilizes the HWIL real-time vector processing techniques. MMW scene generator optimization will be leveraged on this activity.

\section{Conclusion}

The development of the P3I BAT HFS IR and MMW scene generators is ongoing. The methodology of utilizing IR and MMW target models developed with collected measurement data has given the fidelity required to generate realistic imagery of the simulated battlefield. The availability of low-cost high performance computers has allowed for the implementation of real-time scene generation techniques and has increased the commonality between the HFS and HWIL simulation. As development progresses, the HFS will become an even more powerful tool for performance assessment, system design and algorithm development.

\section{References}

1. Kissell, Ann H., Williams, Joe E., Drake, V. Marsha: "Development of an IR/MMW High Fidelity Flight Simulation (HFS) for the P3I BAT Submunition", presented at the American Institute of Aeronautics and Astronautics (AIAA) First Biennial National Forum on Weapon Systems Effectiveness. (1999)

2. TI/Martin Javelin Joint Venture: "JAVELIN Integrated Flight Simulation User's Manual", Version 2.0, Document Number ND-0078. (1993)

3. Kissell, Ann H.: "P3I BAT Simulation Transition Proposal", briefing. (1996)

4. Simulation Technologies: "Simulation Technologies, Inc", briefing. (2001)

5. Saylor, Annie V.: "BAT P3I Target Model Scatterer Number Study", Letter Report, RDSS-99-04. (1999)

6. Saylor, Annie V.: “MMW Background Models for P3I BAT”, Letter Report. (2000) 